Musées, Patrimoine et Culture scientifiques et techniques

$138 \mid 2011$

novembre - décembre 2011

\title{
Regard sur la conservation préventive dans les musées de France
}

Insight into preventive conservation in French Museums

Roland May

\section{OpenEdition}

\section{Journals}

Édition électronique

URL : http://journals.openedition.org/ocim/973

DOI : $10.4000 /$ ocim.973

ISSN : 2108-646X

Éditeur

OCIM

Édition imprimée

Date de publication : 1 novembre 2011

Pagination : 5-10

ISSN : 0994-1908

Référence électronique

Roland May, «Regard sur la conservation préventive dans les musées de France », La Lettre de l'OCIM

[En ligne], 138 | 2011, mis en ligne le 01 novembre 2013, consulté le 01 mai 2019. URL : http://

journals.openedition.org/ocim/973; DOI : 10.4000/ocim.973

Tous droits réservés 


\title{
Regard sur la conservation préventive dans les musées de France
}

\author{
Roland May \\ Directeur du Centre interrégional de Conservation \\ et Restauration du Patrimoine (CICRP) de Marseille
}

2011 : vingt années nous séparent de la prise de conscience de nombre de professionnels de musées français en entendant nos collègues canadiens parler de « conservation préventive » lors des Entretiens Jacques Cartier à Lyon en 1991 et de constater le retard que nous avions face à une autre approche possible pour assurer la pérennisation des biens patrimoniaux ${ }^{(1)}$.

Mais au cours de ces vingt années, le retard a été rattrapé et si chacun définissait la conservation préventive à sa manière ${ }^{(2)}$, la prise de conscience collective du lien étroit entre l'objet patrimonial, ses composantes matérielles et son environnement s'est progressivement établie pour entraîner un questionnement, une attention sur l'objet dans sa « quotidienneté », souvent absente dans les années antérieures.

\section{Les collections replacées au cœur des politiques patrimoniales}

Le constat le plus important est d'avoir replacé au cœur des politiques muséales notamment dans le domaine de la gestion et de l'exploitation des collections (étude, prêt, rénovation, exposition, restauration...), leur conservation. Celle-ci était bien souvent perçue comme une contrainte voire un frein à une politique muséale tournée essentiellement dans les années 1980, pourtant marquées par de grands chantiers muséographiques, vers l'augmentation de la fréquentation, la création d'expositions ou d'évènements, jusqu'à façonner une opposition entre un soi-disant immobilisme de la conservation et la dynamique de la diffusion. Elle devient - elle redevient - à partir des années 1990 un socle - une «bonne pratique du quotidien » - autour duquel peuvent se retrouver professionnels patrimoniaux et décideurs pour élaborer ensemble une politique cohérente et réfléchie de transmission et de diffusion.

La conservation préventive s'est ainsi affirmée car elle n'imposait rien ex cathedra, elle expliquait. Elle apportait deux composantes essentielles à sa crédibilité : une méthodologie dans le cadre d'une vision globale et un appui scientifique ${ }^{(3)}$. Elle permettait de résoudre et d'améliorer des situations à partir de constats, d'argumentaires, grâce à une analyse des objectifs, des paramètres en jeu. La mise en place du projet scientifique et culturel quelques années auparavant, avait ouvert le chemin pour une telle démarche. Source de dialogue et force de proposition, elle se présentait comme un régulateur entre différentes opinions en apportant à chacun des garanties, dans un compromis constructif. La conservation préventive devenait ainsi le médiateur du paradoxe muséal conservation/monstration et participait au débat avec intelligence et clairvoyance.

Elle s'est également imposée car elle véhicule des notions de rationalité, d'économie, de réflexion, d'anticipation, d'adaptation... particulièrement bien entendues à partir des années 1990 lorsque les budgets des puissances publiques commencèrent à se resserrer et que l'euphorie des années 1980 montrait ses limites. Les problématiques qu’elle soulevait, certes complexes et par moment contradictoires, étaient très concrètes et s'exprimaient dans un langage simple induit par la diversité des acteurs - décideurs, services techniques, professionnels de musée, restaurateurs, public...-, à la différence des domaines plus techniques ou spécialisés comme la restauration, le traitement d'air...

Tout un chacun peut comprendre et parler de prévention même si la mise en œuvre est technique et nécessite compétence et professionnalisme.

L'évolution des musées en France depuis les années 1980 - politique de rénovation, de modernisation, de diffusion, professionnalisme, nouveaux acteurs culturels -, a permis à la conservation préventive de faire son chemin à la fois de manière endogène car elle répondait à un besoin de réflexions et de solutions adaptées pour pérenniser techniquement et financièrement les collections, pour mieux les 
gérer et les exploiter, et exogène car la mondialisation patrimoniale avec la circulation d'expositions, la politique de l'évènementiel, confrontaient les professionnels et les institutions françaises aux autres institutions européennes ou nord américaines qui avaient intégré de telles politiques depuis plusieurs années.

Ce dépoussiérage d'un concept pourtant ancien - la conservation - explique certainement que les réserves, le « chantier des collections » sont devenus des projets à part entière, mis en exergue en tant que tels, alors que moins de dix ans auparavant ils seraient passés inaperçus ${ }^{(4)}$. Même des thématiques de conservation, récurrentes depuis les années 1970 comme le climat ou la lumière, ont été revues avec une approche plus dynamique ${ }^{(5)}$, plus constructive, mieux pensée. À ces champs se sont rajoutés d'autres tels que les infestations, les polluants, la poussière, les vibrations, le marquage... (6). Le second facteur de l'implantation de la conservation préventive dans les pratiques muséales est sans aucun doute la mobilisation de l'ensemble des acteurs professionnels pour accélérer sa mise en place et sa pratique :

\section{De la nouvecuité à l'achualité perpétuelle}

Le contexte général de l'évolution muséale des années 1980 à 2010 décrit dans l'article principal, s'est accompagné de mesures, de concepts, de méthodes qui se sont mis en place à partir et à destination des collections.

La focale professionnelle des responsables des collections, des conservateurs - malheureusement pas toujours car plus souvent intéressés par les acquisitions ou les expositions, un peu par les restaurations - des régisseurs et aussi de bien des restaurateurs a pris de la hauteur pour ne plus seulement regarder l'objet en tant que tel mais le replacer dans son environnement, dans son histoire matérielle, dans sa vie quotidienne, dans son usage et son avenir. Elle s'est également portée sur l'objet, les collections dans une vision dynamique d'usage, d'exploitation et de gestion.

Face à ces mutations sont nés (ou ont été remis en lumière) des outils et des notions pour concrétiser ces dynamiques. Ainsi a-t-on vu se développer les réserves et à une place jusqu'alors inédite. Ce phénomène qui est spécifique aux musées à la fin des années 1990 s'est étendu à l'archéologie avec la mise en place de centres de conservation et d'études, inspiré des « pôles de conservation », voulant là aussi provoquer une rupture sémantique par rapport au « dépôt de fouilles » et à quelques images (certes pas générales) de caisses entassées ou de cartons éventrés, et promouvoir à la fois un lieu de stockage en conditions adaptées et un lieu de travail (traitement et études). D'autre part, de nombreuses opérations sur et autour des collections ont été mises en œuvre au début des années 2000 :

- opérations réglementaires avec l'instauration par la loi sur les musées de France du 4 janvier 2002 du rappel de la tenue de l'inventaire et surtout de l'obligation d'un récolement décennal et universel dont l'aboutissement, pour ce premier dispositif, sera 2014 ;

- opérations gestionnaires avec l'apparition dans le monde des musées de la notion de « chantier des collections » dont celui du musée du quai Branly fut le plus emblématique par les moyens mis en œuvre, mais qui eut des précédents plus discrets dont celui - exceptionnel et avant-gardiste - du musée du Conservatoire national des Arts et des Métiers. Celui du Quai Branly fut à l'origine de nombreux autres, encouragés par les puissances publiques, confortées par de nombreuses formations par l'INP, mises en œuvre par des conservateurs-restaurateurs spécialisés en conservation préventive... Ces « chantiers » furent quasi systématiques pour toutes les rénovations de musées et de réserves à partir des années 2000 et s'imposent aujourd'hui dans la plupart des chantiers en cours, le plus important étant aujourd'hui celui du MUCEM dans le cadre du transfert de collections à Marseille.

Enfin, une autre notion a connu et connaît toujours un intérêt d'actualité, celle du plan de sauvegarde ou plan d'urgence qui a pris une ampleur après diverses catastrophes ou incidents comme la tempête de 1999, l'incendie de l'ancien Parlement de Rennes en 1994 et celui du château de Lunéville en 2003. Le risque des crues de la Seine en 2002, crédibilisé par les crues de Dresde et de Prague, a porté à son paroxysme la prévention, et s'est traduit par des incitations à réaliser des plans d'urgence, à modéliser l'évaluation du risque et à stimuler des acteurs comme le comité français du Bouclier bleu.

Si tous ces éléments ne sont pas nécessairement entrés dans la conscience publique, bien que certaines opérations comme le chantier des collections aient fait l'objet de la part des établissements de plans de communication (Valence, Agen), ils font dorénavant partie de l'acquis voire de la pratique de responsables des collections et constituent aujourd'hui moins des sujets d'actualités que des tâches, des missions et des préoccupations du quotidien - ce qui illustre bien que ces concepts, apparus il y a une dizaine d'années, ont été rapidement intégrés et adoptés par la communauté professionnelle de la conservation. L'effort doit être simplement poursuivi car la tâche est longue et surtout constante : à ce titre, elle est perpétuellement d'actualité. 


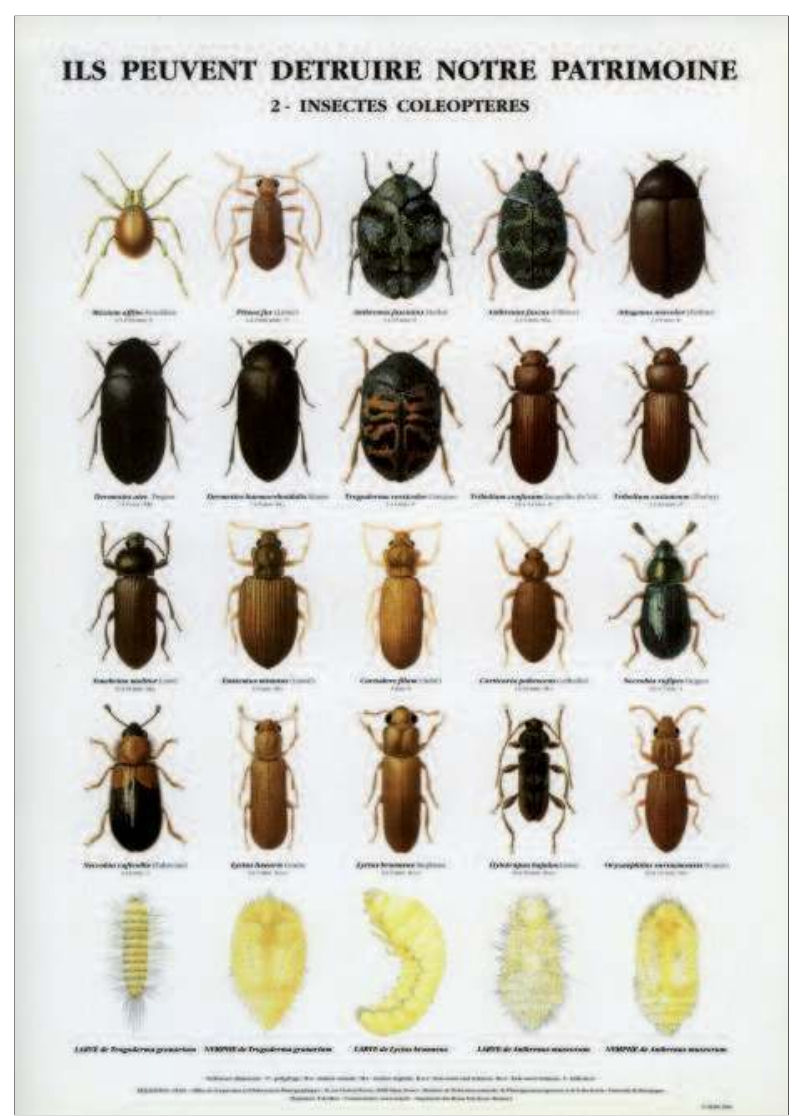

๑) OCIM/G. Pacaud/Dessins F. de Mori

acteurs institutionnels (Direction des Musées de France, Centre de Recherche et de Restauration des Musées de France), acteurs de la conservation (restaurateurs se spécialisant en conservation préventive), création d'équipes mixtes architectes-programmistes-restaurateurs " préventistes », développement de formation initiale (Master de Paris I) ou continue (Institut national du Patrimoine, département des conservateurs) (7). Au-delà du nombre, ces formations ont vu se mêler dans la même assistance des professions et des domaines différents : conservateurs, régisseurs, techniciens..., musées, monuments historiques, bibliothèques, muséums montrant que cette conservation matérielle du patrimoine constitue bien le fondement de tous ces métiers et de toutes ces institutions malgré une histoire, une culture, des moyens voire des objectifs différents (8) et que la voie à prendre doit être interdisciplinaire et transversale.

\section{Peut-on alors au terme de ces vingt ans, face à cette prise de conscience rapide et collective et à un certain succès, conclure à un horizon serein ?}

Il serait d'abord souhaitable de s'assurer que le succès constaté ne soit pas un phénomène conjoncturel, sorte d'effet de balancier aux années 1980, ou résultant de contraintes imposées par les institutions étrangères lors de prêts... Il faudrait donc le mesurer et en tirer des indicateurs et des perspectives.

Il peut certes se percevoir grâce aux chantiers réalisés et à l'expérience acquise confortant les démarches méthodologiques recommandées, les mises en œuvre et les résultats de projet. Mais il serait certainement plus profitable d'établir un bilan des opérations réalisées, analyser le rapport et l'évolution des coûts/budget de conservation/suivi des collections avec celui de la restauration, comparer financièrement et matériellement les politiques des établissements en fonction des pratiques préventives retenues...

Malheureusement, la France ne possède guère cette culture du bilan et de l'évaluation. Ne pourrait-on pas concevoir un observatoire des politiques de conservation-restauration ? La mission parlementaire sur les réserves (9) avait noté en 2002 la carence sur la capacité des instances nationales à donner une vision relativement complète et juste de la situation des collections et des réserves. Elle pourrait probablement la reformuler dix ans plus tard si on devait évaluer la politique de conservation préventive menée depuis 2000. Les rares cas d'analyses et de mise en perspective telle l'enquête expérimentale sur la HauteNormandie sont restés sans suite ${ }^{(10)} \ldots$ ou au stade de concept (Hexaplan).

Des schémas directeurs et des indicateurs pertinents en conservation dans des politiques publiques, comme cela s'est fait aux Pays-Bas à la fin des années 1990 avec le Plan Delta, seraient nécessaires pour crédibiliser un domaine qui relève en grande partie de pratiques quotidiennes et peu visibles.

Les études et la recherche appliquée devraient être développées car l'apport scientifique participe à la crédibilité des préconisations par rapport à des enjeux environnementaux et réglementaires dont l'évolution est guidée par la santé et la sécurité publiques ou par le secteur économique. Le patrimoine a ses spécificités et doit pouvoir bénéficier de considérations et de recherches particulières. La reconnaissance d'une recherche scientifique au service de la conservation, le rôle des « conservation scientists », la diffusion des résultats sont nécessaires au fondement d'une politique et d'une discipline de la conservation. Or ce point est le talon d'Achille de la conservation-restauration en France, rien de comparable par exemple, à l'esprit des travaux menés par l'Institut canadien de Conservation (11).

Ce socle bipode - évaluation/appui scientifique - doit être d'autant plus fort dans les années à venir que l'un des facteurs clés de la réussite des années 2000 à savoir un appui institutionnel conséquent de l'État, est en train d'être réformé. Il faudra voir, en terme de politique et de moyens, si 


\section{Les "réserves »: un nouvecu regard, un nouvel outil}

Il est certain qu'au regard de l'histoire des musées et de la conservation, à la fin des années 1990 et surtout lors de la première décennie du XXIe siècle, les « réserves » auront acquis, en peu de temps, des lettres de noblesse faisant de ces lieux mais aussi de leurs fonctions « mise en réserve » une entité à part entière et non plus le lieu de rebut du «non exposable » ni le lieu de l'oubli.

Cette mutation a essentiellement trois raisons concomitantes, l'une ayant souvent entraîné les autres : le besoin économique et spatial d'externaliser les collections non présentées, processus apparu lors des rénovations de musées, développement de la conservation préventive et prise de conscience de l'impact environnemental en salle comme en réserves et enfin utilisation des objets en réserve pour des animations, expositions, prêts... permettant une activité publique à moindre coût et d'autant plus aisée à conduire que les collections sont bien rangées, identifiées et conditionnées sur des rayonnages...

Ce contexte a ainsi vu se mettre en place de véritables projets de réserves, généralement externalisées, souvent mutualisées avec la redéfinition des modalités de fonctionnement nécessitée par l'éloignement (connexion Internet, accès à l'inventaire, espaces de travail pour une équipe plus présente, organisation du travail et des déplacements), souvent impulsée et réussie grâce à l'arrivée des régisseurs attachés spécifiquement à la vie des collections (inventaire, récolement, modalités de prêt, logistique...).

Cette nouvelle vie, avec ses activités multiples, faisait ainsi de ces lieux bien plus que des « réserves » au point de proposer une notion plus appropriée de "pôle de conservation » qui s'adossait au «pôle de diffusion » qu'était le musée lui-même avec ses expositions et l'accueil du public, ces deux pôles constituant bien entendu les deux piliers de l'institution muséale.

Au-delà du concept et de ce développement, il faut aussi retenir la mise en place d'une méthode de conception de réserves avec, pour tous les projets externalisés mais aussi pour certaines réhabilitations interne de réserves, une place importante accordée, dans le projet scientifique et culturel du musée, aux « réserves » et à la gestion des collections. Parfois même, lorsque l'enjeu est important, il s'agit d'un PSC spécifique comprenant une étude sur les collections (état matériel, diagnostic, préconisations de traitement, calcul de l'encombrement des collections reconditionnées...) conduite par un conservateur-restaurateur spécialisé en conservation préventive -, une étude de programmation incluant l'étude précédente proposant des superficies, des fonctionnalités... et enfin, dans certains cas, un concours d'architecte pour construire ou réhabiliter des lieux pour en faire des réserves, des pôles de conservation de qualité, offrant à la fois de bonnes conditions de conservation et des possibilités de travail aux équipes.

C'est ainsi qu'à partir de la fin des années 1990, se sont mises en place des réserves de grande qualité et dans des villes d'importance très diverse : à Saint-Étienne - première réserve externalisée et mutualisée ayant fait l'objet du processus décrit ci-dessus - Valence, Marseille, Lonsle-Saunier, Montauban, Louviers, Agen, Dijon... processus qui se poursuit avec le centre de conservation du MUCEM en cours de construction à Marseille. Certains de ces chantiers ont même été inaugurés lors de leur lancement ou de leur achèvement conférant à ces lieux pourtant réputés fermés et d'une utilité " publique » relative une place presque égale aux lieux de monstration.

L'actualité du sujet et l'émergence de ce nouvel outil pour mieux gérer les collections a amené un travail français puis européen de normalisation sur ces nouveaux équipements en identifiant les fonctions souhaitables pour assurer une bonne gestion (1).

Les réserves sont devenues un lieu valorisant pour les responsables de collections et même pour les décideurs, probablement parce que la démarche a été très volontariste de la part des puissances publiques et a reçu le soutien des professionnels de la conservation, certainement aussi parce qu'elles ont été présentées comme des outils de gestion et que les notions " patrimoine », « conservation » semblent avoir progressé dans la conscience collective, certes sous formes diverses, notion de durabilité, confrontation monde moderne/traces du passé, support de politique touristique... mais sur un socle commun qui est celui de la conservation des œuvres et des objets patrimoniaux au service des publics et de la population.

\section{Note}

(1) Une norme française est parue en 2007 : XP X 80-001 Décembre 2007, Conservation des biens culturels - Pôle de conservation Définition et caractéristiques fonctionnelles des espaces permettant la conservation et l'exploitation de biens culturels qui préfigure le document européen. Une norme européenne est en cours de préparation : prEN 16141, Conservation of Cultural Property - Open storage facilities : definitions and characteristics of collection centers dedicated to the preservation and management of cultural heritage. 


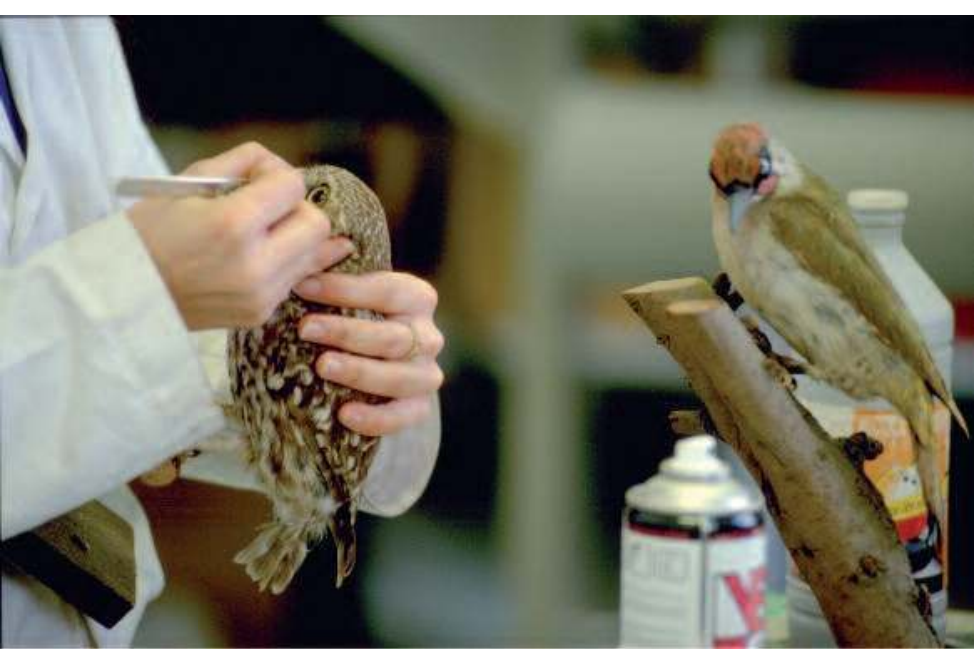

(c) OCIM/François Tomasi

l'impulsion, l'incitation technique ou financière, les préconisations... seront portées de la même manière que par le passé. Si ce n'était pas le cas et l'on peut parfaitement considérer que cette « bonne pratique du quotidien » relève totalement de la politique de l'établissement, il faut que celui-ci puisse se référer, face aux décideurs, à des éléments tangibles et objectifs.

À ce premier enjeu de politique générale en conservation préventive, viennent se greffer assez intimement la sensibilisation et l'éducation à la conservation citoyenne car au-delà des dynamiques institutionnelles ou nationales, l'ancrage dans le quotidien, tant auprès du personnel des établissements, des décideurs que des utilisateurs, est garant du maintien et du développement de ce qui est autant un état d'esprit qu'une discipline. Expositions, conférences, supports didactiques doivent faire prendre conscience de la fragilité du patrimoine. La notion de pérennité doit être constamment réaffirmée dans nos sociétés, notamment auprès des générations actuelles habituées à l'instant et au changement incessant.

Enfin le dernier grand enjeu, probablement le plus innovant, sera la confrontation conservation préventive/développement durable (substanibility). Cette confrontation est double : technique et scientifique comme n'importe quel autre domaine cherchant à trouver les moyens de réduire

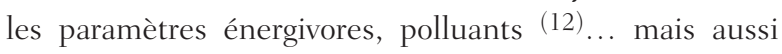
déontologique, de par la spécificité patrimoniale, car à la différence de tout autre objet humain qui peut évoluer ou disparaître par inutilité ou dangerosité, le bien patrimonial conçu à un moment donné de l'histoire et du savoir humain doit être préservé dans son intégrité et dans le temps. Ce constat signifie qu'une procédure de développement durable générale pourrait s'avérer inadaptée à la bonne conservation du bien patrimonial, car elle menacerait, par exemple ses composantes ou son intégrité et qu'une adaptation spécifique de cette procédure doit être faite, cet enjeu justifie une fois de plus des recherches et des préconisations particulières.

\section{Pour conclure}

Le succès de la conservation préventive au bout de ces vingt ans a d'abord reposé sur la nouveauté du regard engendrant une sensibilité accrue à la matérialité de l'objet et à son environnement et un intérêt pour des outils et des procédures : réserves, chantiers des collections, inventaire, récolement, conditionnement, travaux normatifs...Elle s'est révélée aussi être un moyen pragmatique et constructif au service d'un objectif clair : la pérennisation du bien patrimonial.

Elle doit aujourd'hui, pour pouvoir poursuivre cette démarche évolutive, se reposer sur des références opérationnelles et scientifiques lui permettant continuellement d'anticiper, de s'adapter et de se développer au gré des contextes politiques ou socio-économiques.

Et le champ culturel qui reste à investir est encore vaste et divers...

\section{Notes}

(1) Les Entretiens Jacques Cartier n'ont pas fait l'objet d'une publication mais ils furent suivis en 1991 par le congrès de l'ARAFFU avec des actes : La conservation préventive. Paris 8-10 octobre 1992. À lire le n³4 de Technè (automne/hiver 2011) consacré entièrement à la conservation préventive et son implantation notamment institutionnelle en France, réalisé sous la direction de D. Guillemard et R. May.

(2) Lusage de la notion " conservation préventive » a été des fois assez confuse en mélangeant souvent le préventif et le curatif. La notion de conservation-restauration qui s'est progressivement affirmée dans les années 1990 n’a pas toujours facilité le débat, confondant des problématiques de domaines (conservation, restauration), à des enjeux de métier (conservateurs, restaurateurs, conservateurs-restaurateurs, même la notion de « préventiste » avait fait son apparition). Le temps et la réflexion ont fait leur effet avec l'adoption par la communauté professionnelle internationale de définitions au comité de conservation de l'ICOM (ICOM-CC) à New Dehli, 2008 et surtout il est dorénavant possible de se référer à la norme européenne EN 15898 « Conservation des biens culturels - Principaux termes généraux et définitions correspondantes » qui définit « conservation préventive » comme " mesures et actions visant à éviter ou à limiter dans le futur une dégradation, une détérioration et une perte et, par conséquent, toute intervention invasive ».

(3) Cf les articles de Boutaine, J.-L., Dubus, M., Ezrati, J.-J., Féau, É. et May, R. La mise en place d'une politique nationale dans les Musées de France : d'une cellule à un département de conservation préventive et Guillemard, D., May, R. et de Wallens, A. Conservation préventive : conception et structuration d'une pensée, Technè, n³4, 2011. 
(4) Les réserves sont devenues des projets emblématiques et bénéficièrent d'une nouvelle perception les considérant comme les outils premiers d'une bonne conservation : Collections publiques et réserves, Musées et collections publiques de France, n²28, 2000, pp. 229-230 ; Della Monica, A., Deloncle, J., May, R., Valaison, M.-C. et Bertin-Bergston, F. Perpignan, un projet de réserves externalisées et communes. Pour une nouvelle démarche en matière de programmation des collections et de projet de réserves, Technè, $\mathrm{n}^{\circ} 19,2004$, pp. 106-114 ; May, R. Les réserves de musée : nouvelles missions, nouvelles fonctions, nouvelles appellations, Technè, n²1, 2005, pp. 109-112. Il est intéressant de comparer la différence de médiatisation entre le chantier des collections du musée du Quai Branly (2001-2004) et celui, pourtant tout aussi spectaculaire, du CNAM et du transfert des collections à Saint-Denis au milieu des années 1990. De même ce très emblématique chantier des collections du musée du Quai Branly a été la partie émergée d'un iceberg qui avait touché des lieux très divers comme Valence, Agen, Louviers (Portiglia, H., Reibel, L., Boiché, I. et Bergeaud, C. Une opération exemplaire en conservation préventive au musée de Louviers, Technè, n¹7, 2003, pp. 81-88, catalogue d'exposition Dans les coulisses du musée. La conservation préventive des collections. Musée de Louviers 2003).

(5) Ezrati, J.-J. et Sirven, M. La conservation préventive comme données et non comme contrainte. Le cas de l'exposition Rome 1850. Le cercle des artistes photographes du Caffe Greco, Technè, n²1, 2005, pp. 113116.

(6) À titre d'exemple : Dubus, M., Calligaro, T., Ezrati, J.-J., Lambert, E., Melard, N., Perego, F., Pichon, L. et Ravaud, E. Marquage securitaire des biens culturels, Technè, 2007 (Hors série), pp. 75-85; Dubus, M. Guide pratique pour le marquage didentification des biens culturels, version 4.9, 9 C2RMF, août 2010, 13 p. ; Baslé, K., Bouilon, N., Fohrer, F., Guillon, O. et May, R. Pour une approche raisonnée des problématiques d'infestation en milieu patrimonial : le cas du Stegobium paniceum, Technè, n²9, 2009, pp. 109-114 ; Dubus, M., Saraille, S. et de Wallens, A. Transport et climat. L'expérience de dix ans de collaboration avec le département des peintures du Louvre, Technè, n²1, 2005, pp. 117-120 ; Dubus, M. et Laurent, L.-M. Tout ce que vous devez savoir sur les vitrines en bois, Technè, n²9, 2009, pp. 101-108.

(7) Voir note 4.

(8) La loi sur les musées de France (4 janvier 2002, Code du Patrimoine) constitue un dispositif clé dans cette dynamique transversale et globale, fondement même de la conservation préventive, en ayant mis sous une appellation et des pratiques communes les musées du ministère de la Culture, de l'Éducation nationale, de la Défense...

(9) Nachbar, P. et Richert, P. Collections des musées : là où le pire côtoie le meilleur, Sénat, commission des affaires culturelles, mission d'information chargée d'étudier la gestion des collections des musées, Les rapports du Sénat, n³79, 2002-2003.

(10) Le Ray-Burimi, S., Bertin, F., May, R., Bergeaud, C. et Féau, É. Vers un «Plan Delta » en Haute-Normandie : enquête sur l'état et les conditions de conservation des collections des musées de France, Technè, n²3, 2006, pp. 33-39.

(11) Extrait de la page de présentation du site de l'ICC : « La recherche et le développement constituent le fondement même des activités de services et de diffusion des connaissances de l'ICC. Nos activités de recherche sont axées sur les besoins de la communauté du patrimoine au Canada. Nous tenons compte de trois grands critères: la recherche doit avoir une incidence favorable sur la préservation des collections patrimoniales au Canada, la recherche doit avoir une incidence sur l'accès aux collections canadiennes et sur la compréhension de ces collections, les considérations propres à l'Institut ».

(12) À titre d'exemple, les mesures de décontamination de l'amiante ont suscité des interrogations pour des objets manufacturés qui contenaient d'infimes particules d'amiante. La question du désamiantage était-elle nécessaire dans ce cas ? Certes au regard de la réglementation générale mais qu'en est-il au regard de la quantité infinitésimale, comment procéder et qu'en est-il en terme des composantes et de l'intégrité de l'objet ?... Cette question et d'autres de cette nature que l'on rencontre dans le patrimoine industriel, scientifique, naturel se poseront également pour les créations contemporaines dans les décennies à venir (source lumineuse, matériaux...) selon l'évolution des règlementations de santé publique, de sécurité ou de développement durable... 